Comunicación/Educación: escenarios de la definición del campo

Magalí Catino, Margarita Eva Torres, María Gelly Genoud

http://perio.unlp.edu.ar/ojs/index.php/question/article/view/4690

Cita sugerida: Catino, M., Torres, M., y Genoud, M. (2018). Comunicación/Educación: escenarios de la definición del

campo. Question, 1(59), e069. doi:https://doi.org/10.24215/16696581e069

\title{
Comunicación/Educación: escenarios de la definición del
}

\section{campo}

Communication/Education: scenarios of the definition of the

discipline

Magalí Catino magali.catino@gmail.com

http://orcid.org/0000-0002-9402-4809

Facultad de Periodismo y Comunicación Social;

Universidad Nacional de La Plata/

Universidad Nacional de Quilmes (Argentina)

Margarita Eva Torres margameis@yahoo.com.ar

http://orcid.org/0000-0003-3342-8954

Facultad de Periodismo y Comunicación Social;

Universidad Nacional de La Plata (Argentina)

María Gelly Genoud gellygenoud@yahoo.com

http://orcid.org/0000-0001-7911-9837

Facultad de Periodismo y Comunicación Social;

Universidad Nacional de La Plata (Argentina) 


\section{Resumen}

El presente artículo analiza los contextos de emergencia de los profesorados en comunicación en las universidades nacionales de la República Argentina. Pretende rastrear y describir preliminarmente las transformaciones sociales que han repercutido en la configuración del campo Comunicación/Educación.

Los datos que se ofrecen surgen del trabajo que se viene realizando en la investigación "Comunicación/Educación: formación de formadores en comunicación social en universidades nacionales de Argentina. Tendencias y perspectivas". A partir de él es posible delinear algunas de las dimensiones centrales que han dado emergencia a las carreras de profesorados en Comunicación.

Los análisis permiten apreciar que las políticas de corte neoliberal aplicadas en la región, con mayor incidencia en la década de los 90, han impactado en la educación superior afectando diversos factores, particularmente los relativos a la estructuración de los planes de estudio.

Es posible advertir que los cambios que impuso la normativa vigente en cada etapa, así como la demanda por ampliar las posibilidades de inserción laboral de los graduados de licenciaturas y la supremacía de las nuevas tecnologías de la información, son algunos de los factores que han incidido al interior del campo y que son relevados inicialmente en el artículo.

Palabras clave: profesorados; comunicación; educación; universidades; periodización.

Abstract

This article analyzes the emergency contexts of teachers in communication in the national universities of the Argentine Republic. It aims to trace and preliminarily describe the social transformations that have affected the configuration of the Communication/Education field.

The data that are offered arise from the work that is being done in the research. "Communication/Education: training of trainers in social communication in national universities of Argentina. Trends and perspectives". From it it is possible to delineate some of the central dimensions that have given emergence to the careers of teachers in Communication.

The analyzes allow us to appreciate that neoliberal policies applied in the region, with a higher incidence in the 90s, have impacted higher education affecting several factors, particularly those related to the structuring of study plans.

It is possible to notice that the changes imposed by the regulations in force at each stage, as well as the demand to expand the possibilities of job placement of graduates of degrees and the 
supremacy of new information technologies, are some of the factors that have affected to the interior of the field and that are initially surveyed in the article.

Keywords: teachers; communication; education; universities; periodization.

Las carreras de profesorados universitarios en Comunicación se gestan hacia finales de la década de 1990, en buena medida a partir de dos hechos relacionados: por un lado el importante desarrollo de reflexiones y prácticas que tienen como objeto el campo de Comunicación/Educación; y por el otro, la inclusión de contenidos del campo comunicacional como objeto de formación en el sistema educativo formal, que inaugura la por entonces Ley Federal de Educación № 24.195/93 y luego la Ley de Educación Nacional № 26.206/06 en los distintos niveles y a través de diferentes asignaturas.

Es así que las carreras se han ido configurando en las universidades nacionales atendiendo a expresar y recuperar esta doble articulación. Aludimos al campo de formación y de intervención que define el complejo y amplio territorio de trayectorias y prácticas de Comunicación/Educación, así como también a la práctica docente en procesos de formación en comunicación, tanto en espacios de educación popular y en organizaciones, como en el sistema educativo formal (que se inauguraba en el período indicado).

Las transformaciones sociales que se producen en cada época impactan profundamente en los rasgos de lo educativo. Por eso, conocer los antecedentes que fueron marco del surgimiento de los profesorados en Comunicación en distintas universidades del país, es un modo de comprender las necesidades y demandas sociales que se han manifestado en el campo educativo, en particular en lo que a educación superior se refiere.

Al analizar el impacto que las transformaciones sociales, políticas, culturales y económicas que la década de 1990 tuvo en las universidades y en la educación superior en general Catino, Morandi y Ros, refieren que:

Se han ido planteando estrategias que configuraron transformaciones progresivas en el sistema de ES, no sin generar conflictos institucionales y sectoriales aún en debate: nuevas modalidades de gestión, coordinación, financiamiento y evaluación; mecanismos sistemáticos de evaluación y acreditación de las instituciones; y nuevas articulaciones con el sector productivo y el Estado (contratos a terceros, transferencia tecnológica, creación de universidades-empresas, entre otras). En lo relativo a las reformas curriculares, éstas se 
caracterizaron por promover carreras de grado más cortas, títulos intermedios, planes de estudio flexibles, mayor diversidad de orientaciones y especializaciones (Catino, Morandi y Ros, 2005: 104).

Desde hace varios años, la comunicación, como campo de saberes, atraviesa una zona de debate y reflexiones epistemológicas que la obligó a ampliar su objeto más allá de los medios de comunicación, para atender, por ejemplo, a las implicancias de la expansión de las nuevas tecnologías que no sólo configuran nuevas formas de comunicación, sino también nuevos lenguajes y modos de percepción del mundo.

Como cualquier proceso social situado históricamente, las carreras universitarias son atravesadas por las condiciones externas y los licenciados en Comunicación, egresados de las distintas universidades públicas del país experimentaron, en determinados contextos, la necesidad de ampliar sus posibilidades de inserción laboral, especialmente a través de la docencia.

Ese desplazamiento contribuyó además a que fuese conformándose e institucionalizándose el ámbito de Comunicación/Educación, recuperando y articulando nociones de ambos campos de saberes. Como sostiene Da Porta, se trata de una perspectiva donde no se trabaja desde lo interdisciplinario

$\mathrm{Ni}$ pretende unificar ambas disciplinas en un objeto específico, sino que reconoce la pertinencia de una mirada que orada las fronteras disciplinarias y trabaja en el abordaje transdisciplinar de problemas a partir del intercambio crítico de puntos de vista teóricos y metodológicos entre ambas disciplinas (Da Porta, 2004: 70).

La necesidad de los licenciados de generar nuevas opciones laborales se conjugó con las transformaciones percibidas en la esfera de la comunicación, revolucionado por las condiciones socio-históricas y la proliferación de las nuevas tecnologías que modificaron sustancialmente sus prácticas y multiplicaron sus posibilidades, poniendo en tensión sus postulados epistemológicos.

Esas tensiones no se produjeron de manera silenciosa al interior de las universidades, sino que, en muchos casos, se tradujeron en acciones directas y negociaciones ríspidas. Como se ha señalado, estas circunstancias se suscitaron en el marco de un fuerte debate respecto de la comunicación como campo de conocimiento y formación universitaria. Consideramos que es necesario conocer esos procesos y sus derivaciones para contar con un panorama general respecto del surgimiento y transformaciones de la carrera del profesorado en comunicación 
social en el país, sobre todo dada la escasa información de que se dispone, para describir y analizar las transformaciones y realidades de la formación universitaria en comunicación.

La investigación que se viene desarrollando "Comunicación/Educación: formación de formadores en comunicación social en universidades nacionales de Argentina. Tendencias y perspectivas", nos permite presentar algunas de las dimensiones centrales que han dado emergencia a las carreras de profesorados en Comunicación. Los análisis realizados permiten apreciar que las políticas de corte neoliberal aplicadas en la región, con mayor incidencia en la década de 1990, han impactado en la educación superior afectando diversos factores, pero particularmente los relativos a la estructuración de los planes de estudio en base a reformas curriculares que tendieron a la formación de profesionales competentes, especializados, abiertos a la innovación tecnológica, con un fuerte sesgo hacia la productividad y la eficiencia en la posterior inserción laboral en un mercado de trabajo definido por los mismos rasgos.

A partir de poner en diálogo el material relevado en entrevistas recientes con referentes de diversos profesorados en Comunicación del país, y el análisis de las condiciones de su creación, intentaremos delinear una periodización tentativa de las demandas particulares hacia los profesorados en comunicación que entendemos impactaron en la conformación del campo de la Comunicación/Educación.

\section{Demanda laboral: Uno de los factores que incide en la constitución del profesorado}

En la Universidad Nacional del Centro de la Provincia de Buenos Aires (UNICEN) se pensó al profesorado como bloque pedagógico en 1996, en un contexto en el que existían demandas por parte de los graduados de las licenciaturas para acceder a la carrera docente, luego de la sanción de la Ley Federal de Educación. Así, la universidad lo organizó a partir de la constitución de un bloque pedagógico que se incorporaba al trayecto de la licenciatura con materias específicas. Desde entonces, la licenciatura comparte con el profesorado un ciclo común. Según información que nos suministró la casa de estudios, muchos de los estudiantes que comienzan la carrera lo hacen al terminar el secundario y otros la toman como segunda opción, cuando ya están cursando el tramo de la licenciatura. Es interesante señalar que en el caso de que finalicen una, la mayoría de los estudiantes optan por el profesorado, porque les permite una inserción laboral más rápidamente. De hecho, muchos acceden a puestos de trabajo incluso mientras se encuentran cursando el profesorado, antes de graduarse. En UNICEN, en 2014 se cambió el plan de estudios de la licenciatura, pero no el del profesorado. 
En tanto, en la Universidad Nacional de Cuyo (UnCuyo) las consecuencias de las políticas neoliberales aplicadas en los años 90 también incidieron fuertemente en el surgimiento de la carrera. La falta de trabajo de los egresados de las licenciaturas hizo que gran parte de ellos buscaran una salida en la docencia. En ese contexto, los graduados de la Facultad de Ciencias Políticas comenzaron a demandar a la institución para que se abriera la oferta de la formación pedagógica. La existencia de un profesorado en ciencias sociales que existió en esa casa de estudios entre 1974 y 1976 que fuera cerrado durante la dictadura cívico-militar, junto con la licenciatura en Sociología, fue decisiva para que el reclamo por su restauración cobrara fuerza. Según las fuentes consultadas, la lucha fue denodada porque el objetivo de los graduados era contar con un profesorado gratuito y no pago, como ocurría por aquel entonces en Filosofía y Letras. Los graduados debieron recurrir a acciones directas, como la toma de la facultad durante una semana para que, finalmente, en 2002, se creara el profesorado en comunicación social. La carrera Ciclo de Profesorado ofrece cuatro titulaciones y está armada en tres trayectos: el trayecto de la formación general; el trayecto de formación específica y el trayecto de didáctica y práctica. Según resolución de ese mismo año, se estableció que sólo pueden acceder al ciclo los graduados de las cuatro licenciaturas que se dictan en la unidad académica. Otro dato saliente del proceso de UNCuyo es que en 2008, producto de la demanda de personas que venían de otras universidades, se realizó un cambio en el plan de estudios y se abrió el profesorado para las cuatro licenciaturas para egresados de las universidades nacionales. Así, hoy pueden cursar estudiantes de la licenciatura de la Universidad de Buenos Aires (UBA) o de Trabajo Social de la Universidad Nacional de San Juan (UNSJ), por citar algunos ejemplos. En UnCuyo también es preciso ser licenciado o contar con el $85 \%$ de las materias de la licenciatura para acceder al profesorado.

A su vez, en La Universidad Nacional de Entre Ríos (UNER), la carrera de Comunicación surge en 1980, durante la dictadura militar. Se denominó Ciencia de la Información y ofrecía una formación muy instrumental e incluso con una dimensión religiosa. Con el advenimiento de la democracia se hizo una propuesta para modificar el plan de estudios con una mirada más vinculada al nuevo orden mundial de la comunicación y pensarla al servicio de la sociedad. También tuvo fuerte impronta el hecho de considerar las desigualdades que existían entre los países desarrollados y no desarrollados, y la necesidad de trabajar de manera autónoma para construir la comunicación. Siguiendo esa línea, se introdujeron materias como comunicación alternativa, periodismo nacional e internacional, nuevo orden mundial de la comunicación. El plan de estudios fue modificado en 1985 y por última vez en 1998. Como en otros casos, también es requisito ser licenciado para poder hacer el profesorado. 
En la Universidad Nacional de Rosario (UNR) existen dos profesorados. El primero se gestó en 1997 por la demanda de la formación docente a partir de la reforma de la Ley Federal de Educación. Se trata del profesorado en Comunicación Social al que pueden acceder los graduados de la licenciatura.

Durante algunos años se presentaron varios proyectos que apuntaban a tener un profesorado específico en comunicación. Fue así como, en 2014, se aprueba e implementa la carrera de profesorado en Comunicación Educativa. Actualmente, la UNR ofrece el profesorado en Comunicación Social y el profesorado en Comunicación Educativa, cuya primera cohorte egresará este año. Se trata de un profesorado con materias específicas del campo de la educación y la comunicación, considerado una innovación por sus impulsores. En la actualidad conviven los dos profesorados; los que egresan de la licenciatura pueden hacer la formación docente complementaria de dos años, mientras que el profesorado en Comunicación Educativa es una carrera en sí misma, independiente de la licenciatura.

\section{Profesorados en Comunicación: esbozo inicial para su periodización}

A partir de los datos recabados intentaremos ofrecer una periodización de las demandas particulares que consideramos incidieron en la constitución y configuración de los profesorados en comunicación.

Fines de los años 90

Hasta los años 90 no se relevan experiencias significativas en profesorados en Comunicación. Recién a mediados de la década la reforma educativa modifica las modalidades y orientaciones del secundario y la creación de asignaturas específicas oficiará como un incipiente impulso para la constitución de los primeros profesorados en el área. Como señalan Gabriela Gamberini, Stella Pasquariello y María Eugenia Gaite al analizar el caso de la UNICEN:

Recién a finales de los años 90, en pleno auge de las políticas neoliberales y de reformas estructurales y en plena vigencia de la Ley Federal de Educación N24195/93 la formación docente se abre para un conjunto de disciplinas que tradicionalmente no ofrecían carreras de profesorados (2016: 3 ).

En este contexto, la educación es entendida como una práctica social y política con un fuerte potencial transformativo (Gamberini, Pasquariello y Gaite, 2016). 
Podemos consignar que en los años 90, al institucionalizarse la Comunicación como objeto de enseñanza y saber especializado para ser enseñado, se acelera un proceso de profesionalización del campo comunicacional (Farías, 2016).

Las experiencias de la UNICEN en Olavarría en el año 1996 y de la Universidad Nacional de La Plata (UNLP) en el año 1998, serán claves para el devenir de los profesorados en Comunicación en el resto del país. Cabe destacar que en este período también aparecen algunas propuestas de profesorados en Comunicación en institutos terciarios en diversos distritos del país sin vigencia en la actualidad.

En esta etapa surge la carrera en la Universidad Nacional de Rosario (que requiere ser egresado de licenciatura) y también en la Universidad Católica de Santiago del Estero (UCSE).

Por la existencia de estos datos, es que sostenemos que la demanda laboral es uno de los factores que daría impulso a la constitución del campo de la Comunicación/Educación y a su institucionalización a través de la creación de los profesorados.

Después de 2001

En este período irrumpe con más fuerzas la cuestión laboral para tensionar el campo. La necesidad de ampliar el espectro de inserción de los egresados de las licenciaturas aparece en algunos casos como el fundamento central en los planes de estudio de los profesorados. La necesidad del título docente habilitante se torna una restricción de mercado, y se argumenta que los profesores de otras áreas son quienes acceden a los cargos disponibles, dejando por fuera a los formados en Comunicación por carecer de un título docente.

Bajo esta demanda se aprobarán los planes de estudio de los profesorados de la Universidad de Buenos Aires en 2003 y de la Universidad Nacional de Entre Ríos en 2004. En esta última se exige ser graduado de la licenciatura para acceder al título docente. También se constituye el profesorado en la Universidad Nacional del Comahue (UNComa) y en UNCuyo.

\section{Después de 2010}

En esta etapa, los planes de estudio de los profesorados se verán atravesados por una demanda en torno a las tecnologías/tecnicidades y una preocupación pedagógica revitalizada. La inserción laboral perderá centralidad en las propuestas generales aunque nunca será abandonada.

También en ese contexto se consolida una mirada que otorga un lugar central al Estado en la inclusión educativa de los sectores vulnerables de la sociedad. En esa línea, los marcos legales en materia educativa que se establecieron desde 2006, entienden a la educación como 
un bien social y público que contribuye a lograr una mayor justicia social y a superar las desigualdades en el marco de procesos de fragmentación educativa (Gamberini, Pasquariello y Gaite, 2016).

En este período se observa una transformación profunda de la sociedad, donde la expansión de las Tecnologías de la Información y la Comunicación (TIC) han generado nuevas sociabilidades, es decir nuevas formas de relaciones y nuevos vínculos interpersonales. Se redefine el espacio público, como así también lo local y lo colectivo. Lo cierto es que la emergencia de las nuevas tecnologías genera nuevos usos y sentidos de las prácticas educativas tradicionales, demandando nuevas competencias al interior de los procesos formativos de los profesores en Comunicación.

Este nuevo escenario social inaugura un período de redefiniciones en el campo de la Comunicación/Educación, que aún se encuentra abierto. Las TIC operan profundas transformaciones en la concepción del espacio y del tiempo junto con una reorganización de la relación con los saberes.

La educación tradicional había propuesto una relación con el saber mediada por el maestro, centralizada en el curriculum y el libro. Las TIC subvierten esa relación ya que el circuito de circulación, producción, consumo y usos se abre multidimensionalmente. Una serie novedosa de posibilidades creativas y cognitivas aparecerán en este contexto, tales como revisar indefinidamente (iteración), la retroalimentación en el despliegue del proceso de trabajo, la integración de modos distintos de autoría como video, palabra y audio (convergencia), la posibilidad de desplegar el trabajo en distintos formatos y plataformas para distintas audiencias (exhibición) (Dussel y Quevedo, 2010).

La sistematicidad y la formalidad de la educación tradicional, parece entrar en crisis en los tiempos de las TIC. Las nuevas tecnologías basadas en la personalización y la intensidad emocional imponen la necesidad de replantearse la educación en su conjunto.

Todo ello se opone al modo más clásico de relación con el saber que configura la escuela basada en la distancia, la reflexión intelectual, la moderación y hasta la represión de los aspectos emocionales, y el control y reducción de los estímulos visuales más espectaculares, a los que se juzga distractores y poco interesantes (Dussel y Quevedo, 2010: 28).

Los profesorados en Comunicación de este período enfrentan el desafío de pensar la manera de potenciar los procesos de enseñanza a partir de repensar el vínculo con el conocimiento que introducen las TIC. 
Desde la sanción de la Ley de Educación Nacional en 2006 las TIC se incorporaron al contenido formativo obligatorio. Para el 2010, la mirada hacia las TIC que se consolida pone el acento en el impacto sociocultural de los fenómenos comunicacionales que transforman los vínculos con el conocimiento, dejando de lado la necesidad concepción del saber técnico instrumental.

En este escenario se inaugurarán nuevos profesorados como el caso de la Universidad Nacional de Córdoba (UNC) en 2010, el de la Universidad Nacional de Lomas de Zamora (UNLZ) en 2012 y el de la Universidad Nacional de Quilmes (UNQ) en 2015. Conjuntamente se observa la reformulación de planes de estudios como los casos de la UNR en 2014 con el Profesorado en Comunicación Educativa, y la reforma del plan de estudios del profesorado de la UNLP en 2017.

Para visualizar mejor el panorama en relación a la aparición de los profesorados en Comunicación, consignamos que:

Desde 1994 (Ley Federal de Educación) hasta el 2000, se crean cuatro profesorados:

- UNICEN: 1996

- UNR: 1997

- UNLP: 1998

- UCSE: 2000

Después del 2001, se crean cuatro profesorados:

- UNCuyo: 2002

- UNComa: 2002

- UNER: 2003

- UBA: 2004

Desde 2010 hasta la actualidad, en plena vigencia de la Ley de Educación Nacional, se crean cuatro profesorados y se reforma un plan de estudio:

- UNC: 2010

- UNLZ: 2012

- UNR: 2014 (crea una nueva modalidad, el profesorado en Comunicación Educativa y mantiene en vigencia el profesorado creado en 1997 que requiere ser egresado de la licenciatura).

- UNQ: 2015

- UNLP: 2017 (reforma el plan de estudio).

Se puede observar que actualmente en la República Argentina existen once profesorados en comunicación. Diez de ellos se dictan en universidades nacionales públicas y uno en una 
universidad privada. La variedad de titulaciones que ofrecen permite inferir ciertos postulados epistemológicos/teóricos, singularidades de la práctica y cierta definición del campo laboral. Así, por ejemplo, existen profesorados en Ciencias de la Comunicación; profesorados en Comunicación Educativa y profesorados en Comunicación Social.

En todos los casos se evidencia la expansión del campo y la institucionalización de la Comunicación/Educación, la demanda y ampliación del campo profesional del graduado, las transformaciones normativas en educación formal y las tecnologías como vector histórico de transformaciones sociales, políticas y subjetivas.

\section{Bibliografía}

Catino, M., Morandi, G. y Ros, M. (2005) Tendencias en la Educación Superior. Desafíos para la formación en comunicación en un escenario de redefiniciones. Revista Redefiniciones, 103 -109.

Da Porta, E. (2004). Senderos y recorridos. Apuntes para un mapa de investigación. Tram[p]as de la Comunicación y la Cultura, (29).

Dussel, I. y Quevedo, L. A. (2010). Educación y nuevas tecnologías: los desafíos pedagógicos ante el mundo digital. Buenos Aires: Santillana.

Farías, M. (2016). Escuelas Medias Técnicas, educando en comunicación. Los casos de "EET 625 Carlos Guido y Spano" y el "Complejo Educativo No 394 Dr. Francisco de Gurruchaga" (Tesina de grado). Universidad Nacional de Córdoba, Córdoba.

Gamberini, G., Pasquariello, S. y Gaite, M. E. (2017). Aportes para pensar la reformulación de los planes de formación de carreras de profesorados. En Giordano C. y Morandi G. (Comp.), Memorias de las 1a Jornadas sobre las Prácticas Docentes en la Universidad Pública "Transformaciones actuales y desafíos para los procesos de formación". Relatos de experiencias. Reseñas de investigación. Universidad Nacional de la Plata, La Plata.

Recuperado

de: http://sedici.unlp.edu.ar/bitstream/handle/10915/60899/Documento_completo__.pdfPDFA.pdf?sequence $=1$ 